\title{
Does lumbosacral spina bifida arise by failure of neural folding or by defective canalisation?
}

\author{
ANDREW J COPP AND FRANCES A BROOK \\ From the Medical Embryology Laboratory, Imperial Cancer Research Fund, Developmental Biology Unit, \\ Department of Zoology, University of Oxford, South Parks Road, Oxford OX1 3PS.
}

SUMmARY The aim of this study was to determine whether open lumbosacral spina bifida results from an abnormality of neural folding (primary neurulation) or medullary cord canalisation $\stackrel{N}{\sigma}$ (secondary neurulation). Homozygous curly tail ( $c t)$ mouse embryos were studied as a model $\omega$ system for human neural tube defects. The rostral end of the spina bifida was found to lie at the $\vec{g}$ level of somites 27 to 32 in over $90 \%$ of affected $c t / c t$ embryos. Indian ink marking experiments 을 using non-mutant embryos showed that the posterior neuropore closes, and primary neurulation $\rightarrow$ is completed, at the level of somites 32 to 34 . Since neurulation in mammals progresses in a 3 craniocaudal sequence, without overlap between regions of primary and secondary neurulation, $\frac{\infty}{\partial}$ we conclude that spina bifida in $c t / c t$ embryos arises initially as a defect of primary neurulation. The position of posterior neuropore closure in human embryos is estimated to lie at the level of $\mathscr{\infty}_{\infty}$ the future second sacral segment indicating that in humans, as in the $c t$ mouse, lumbosacral spina ${ }^{\oplus}$ bifida usually arises as a defect of posterior neuropore closure. Cranial NTD affect females predominantly, whereas lower spinal NTD are more common in males, both in humans and $c t \xi$ mice. We offer an explanation for this phenomenon based on (a) differences in the effect of embryonic growth retardation on the likelihood that an embryo will develop either cranial or $\frac{\mathscr{\nu}}{D}$

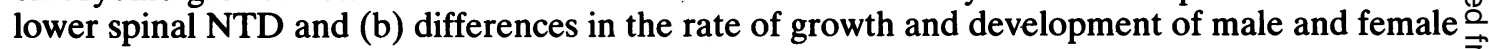
embryos at the time of neurulation.

Neural tube defects (NTD), anencephaly and spina bifida, differ markedly in their sex incidence. Anencephaly affects predominantly females whereas spina bifida, taken as a whole, has a sex ratio near to unity. ${ }^{1}$ One possible explanation for this phenomenon is that the two types of NTD arise through different embryological mechanisms, one being especially susceptible to disturbance in females while the other is not. Seller ${ }^{2}$ suggested that NTD affecting the head or thorax or both, which show a preponderance of affected females, arise through disturbance of primary neural fold closure. On the other hand, post-thoracic defects, in which males predominate, were seen as arising through abnormality of a different embryological process: socalled secondary neurulation. ${ }^{2}$

'Primary neurulation' refers to the process of neural folding whereby the entire brain and much of the spinal cord is formed in mammals. In the spinal region, neurulation proceeds in a craniocaudal

Received for publication 8 July 1988 . Accepted for publication 19 August 1988. sequence from the future cervical region and is completed with closure of the posterior neuropore.0 Neural tube formation at more caudal levels occurs $\frac{0}{3}$ by 'secondary neurulation' in which the neural: primordium, the medullary cord, becomes canalised directly without previous formation of neural folds. ${ }^{3-6} \gtreqless$ There is no region of overlap between primary and $\mathrm{O}$ secondary neurulation in mammals, ${ }^{6}$ unlike the $D$ chick embryo. $^{7}$

In the present study we have examined the mechanism of development of spinal NTD in the . curly tail $(c t)$ mutant mouse which has been much $\tilde{O}^{0}$ studied as a model for human NTD. ${ }^{8-10}$ One striking N similarity between NTD in $c t / c t$ mice and in humans is the pattern of sex ratio distortion: exencephaly the predominant cranial NTD in $c t / c t$ mice, occurs $\widetilde{\Phi}$ mainly in females, whereas open lumbosacral spina $\stackrel{?}{?}$ bifida and tail flexion defects, taken together, have 0 an approximately equal sex incidence. ${ }^{8}$ It is not ${ }^{-}$

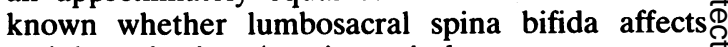
mainly males in $c t / c t$ mice as in humans.

Scanning electron microscope observations early in the process of neurulation show that cranial NTD 
arise in $c t / c t$ embryos by failure of neural fold elevation or fusion. ${ }^{11}$ Studies of $c t / c t$ embryos during spinal neurulation indicate that open lumbosacral spina bifida and tail flexion defects also arise by abnormal neural fold closure. ${ }^{12}{ }^{13}$ These observations suggest that both cranial and lower spinal NTD can arise in $c t / c t$ embryos by a defect of the primary neurulation mechanism. However, previous studies of spinal NTD pathogenesis in the curly tail mutant have been confined to early stages in the development of the malformations and, therefore, cannot exclude the possibility that an additional subgroup of embryos develop spinal NTD later in gestation as a result of defective secondary neurulation, as suggested for the human embryo. ${ }^{2}$

The purpose of the present study was, first, to ask whether lumbosacral spina bifida is associated with male $c t / c t$ embryos as in humans and, second, to find the precise axial level of spina bifida in $c t / c t$ mouse embryos at various stages of gestation, in order to determine whether this malformation arises by a defect of primary or secondary neurulation.

\section{Materials and methods}

\section{MOUSE STRAINS}

The curly tail $(c t)$ mutation was established originally on the CBA/Gr genetic background. ${ }^{14}$ Curly tail mice are now maintained as a closed colony of $c t / c t$ homozygotes. Spinal NTD develop in approximately $60 \%$ of homozygotes: two-thirds of the abnormal mice manifest tail flexion defects (that is, curly tails) and one-third have lumbosacral spina bifida with, in most cases, curly tails. ${ }^{812}$ The remaining $40 \%$ of $c t / c t$ mice are morphologically normal. Experimental litters were obtained from matings between affected males and either affected or unaffected females (all $c t / c t)$.

Mice of the albino PO strain (Pathology, Oxford) served as a source of non-mutant embryos for Indian ink marking experiments.

\section{DETERMINATION OF EMBRYO SEX AND}

ASSESSMENT OF SPINA BIFIDA

A number of curly tail conceptuses were removed from pregnant females on day 13 of gestation (day $1=$ day of finding a copulation plug), extraembryonic membranes were removed, and embryos were inspected in the living state for the presence of exencephaly (the rodent form of anencephaly), spina bifida, or tail flexion defect. The sex of these embryos was determined by gonad morphology as described previously. ${ }^{15}$

In a separate experiment, embryos were removed from curly tail females at various times on days 11 , 12 , and 13 of gestation and those with open lower spinal neural tubes were fixed in Bouin's fluid. These embryos were inspected under a Wild dissecting microscope for somite number (method of Copp et $a l^{12}$ ), somite level of the rostral and caudal ends of the spina bifida, and presence of a tail flexion defect.

\section{CULTURE AND INJECTION OF EMBRYOS WITH} IN DIAN IN K

Non-mutant PO embryos were explanted early on day 10 of gestation into alpha modification of Eagle's Minimal Essential Medium (Flow Labs, UK) containing $2 \mathrm{mmol} / \mathrm{l}$ glutamine, $20 \mathrm{mmol} / \mathrm{l}$ Hepes, $100 \mathrm{U} / \mathrm{ml}$ penicillin, $100 \mu \mathrm{g} / \mathrm{ml}$ streptomycin, and $10 \%$ fetal calf serum. Embryos with prominent forelimb buds have approximately 26 or more somites and these were selected for ink injection. The yolk sac and amnion were opened over the dorsal aspect of the embryo, at the level of the forelimb buds, using watchmaker's forceps. A hand held, mouth controlled micropipette was inserted through the surface of the embryo and into the lumen of the neural tube, with its tip pointing towards the caudal end of the embryo. A solution of sonicated Indian ink (TGI, Higgins), previously dialysed (molecular weight cutoff 12000 to 14000 ) at $4^{\circ} \mathrm{C}$ against phosphate buffered saline, $\mathrm{pH} \mathrm{7.4,}$ was injected into the neural canal. In the majority of embryos, ink spread immediately along the neural canal and emerged from the open neuropore into the amniotic cavity. In a few cases, the ink did not emerge from the posterior neuropore indicating that primary neurulation was already complete. Only embryos with open neuropores were cultured for a further 24 hours, using the method of New et al. ${ }^{16}$ Then the yolk sac and amnion were removed and embryos were inspected for the presence of an ink mark in the roof plate of the neural tube. Somites were counted and embryos were fixed in Bouin's fluid and caudal regions with ink marks were

TABLE 1 Sex incidence of NTD in ct/ct embryos.

\begin{tabular}{|c|c|c|c|c|}
\hline & $\begin{array}{l}\text { Normal } \\
\text { spine }\end{array}$ & $\begin{array}{l}\text { Tail defect } \\
\text { only* }^{*}\end{array}$ & $\begin{array}{l}\text { Spina bifida } \\
\text { and tail } \\
\text { defect }^{*}\end{array}$ & Total \\
\hline \multicolumn{5}{|l|}{ No exencephaly } \\
\hline Male & 158 & 115 & 27 & 300 \\
\hline Female & $111^{1.42 \dagger}$ & $134^{0 \cdot 86}$ & $21^{1.29}$ & 266 \\
\hline With exencephaly & & & & \\
\hline Male & 0 & 0.07 & 0 & 1 \\
\hline Female & 3 & 14 & 7 & 24 \\
\hline Total & 272 & 264 & 55 & 591 \\
\hline
\end{tabular}

*Sex ratio of spinal NTD combined (no exencephaly)=142 males: 155 females $=0.92$.

+ Values are male/female sex ratios. 
prepared as serial transverse histological sections of $6 \mu \mathrm{m}$ thickness.

\section{Results}

SEX INCIDENCE OF NTD IN $C T / C T$ EMBRYOS Eighty $c t / c t$ litters yielded a total of 591 embryos for which gonadal sex was determined. There was a strong female preponderance among exencephalic embryos irrespective of whether spinal NTD were also present (table 1). Considering embryos without exencephaly, the overall sex incidence of embryos with both types of spinal NTD was close to unity (sex ratio 0.92), thus confirming the finding (sex ratio 0.93) of Embury et al. ${ }^{8}$ However, among embryos with spinal NTD and no exencephaly, males were more often affected by spina bifida (sex ratio 1.29$)$ whereas females more often had tail flexion defects alone (sex ratio $0 \cdot 86$ ). Thus, the most severe form of spinal NTD in $c t / c t$ mice, lumbosacral
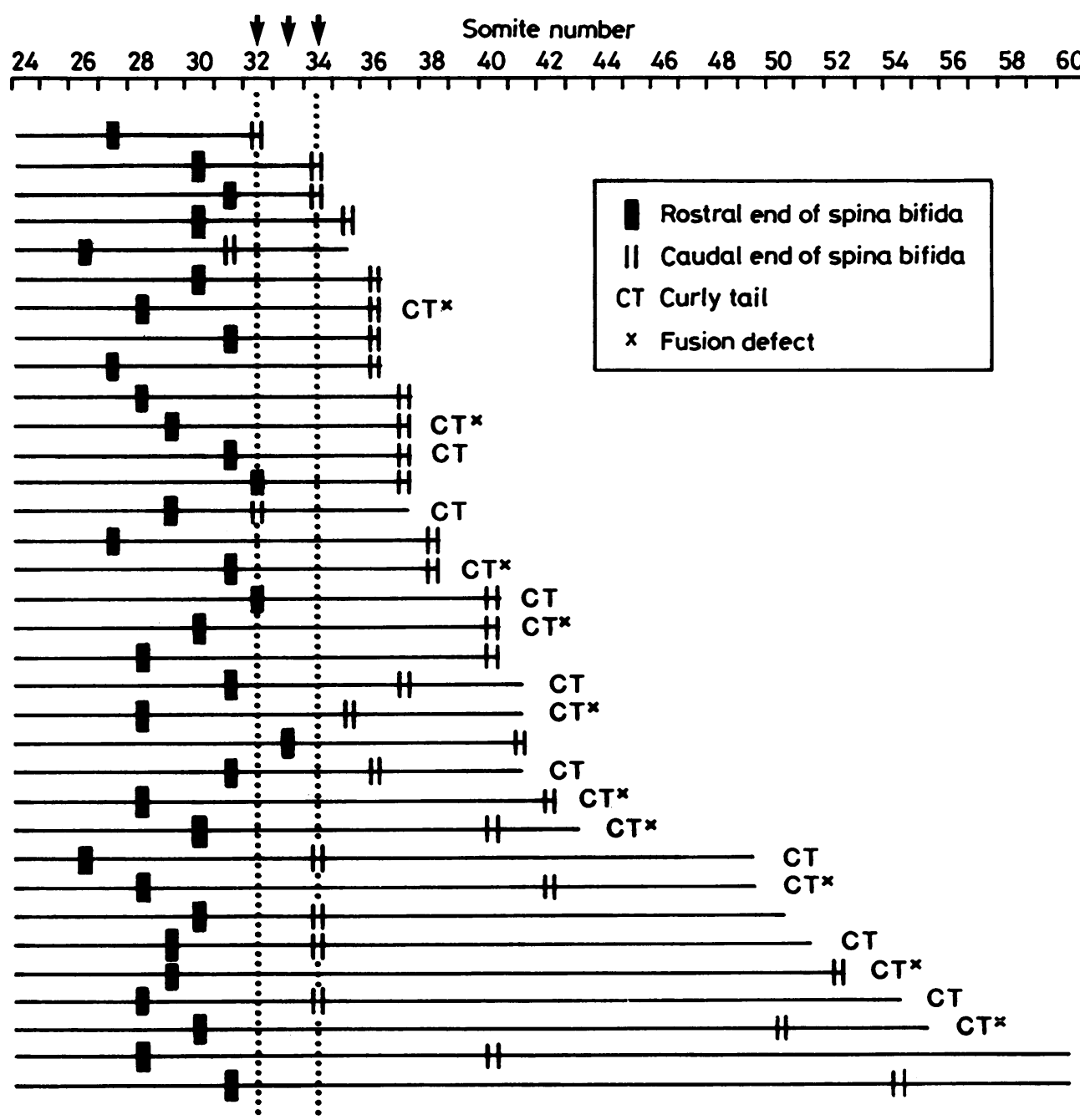

FIG 1 The extent of open spina bifida in ct/ct embryos. Each horizontal line depicts the caudal portion of a single embryo. The somite stage of the embryo is indicated by reference to the scale at the top of the diagram. For each embryo, solid black vertical bars show the somite level of the rostral end of the spina bifida and vertical paired lines show the caudal limit of the spina bifida. CT indicates the presence of a tail flexion defect. ${ }^{{ }}$indicates the presence of a proximodistal fusion defect (see text for explanation). Arrows at the top of the diagram and vertical dotted lines show the position of closure of the posterior neuropore, as determined in the Indian ink marking experiment. 

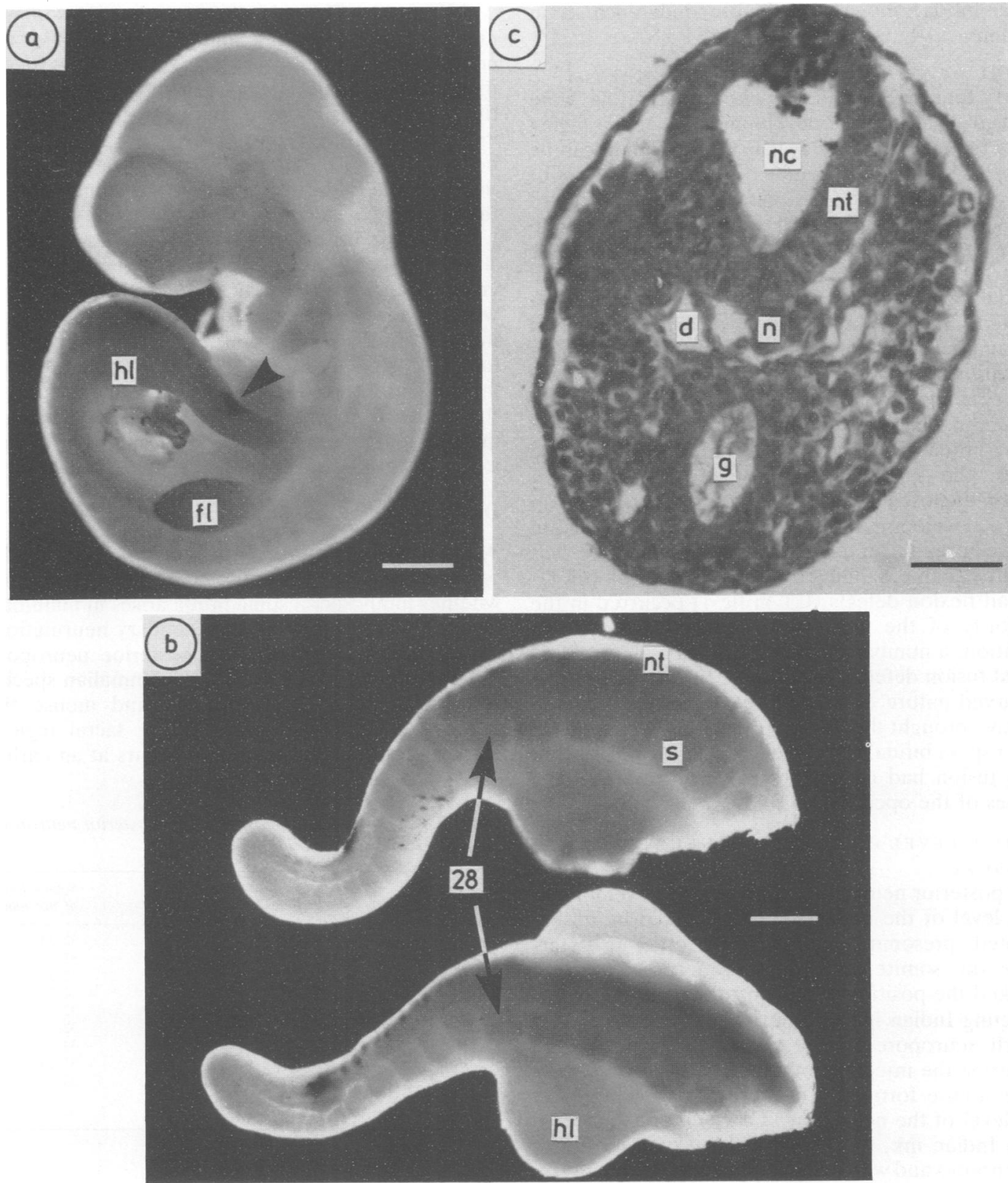

FIG 2 Non-mutant PO embryos that received an injection of Indian ink into the neural canal and were subsequently cultured for 24 hours. (a) Whole live embryo after culture exhibiting prominent ink mark in the dorsal aspect of the neural tube (arrowhead). (b) Caudal regions dissected from two cultured embryos, after fixation, to illustrate the somite position of ink marks in the neural tube roof. Counting from the 28th somite (arrowed), the ink marks are opposite somites 33 to 34 in the upper embryo and somite 34 in the lower embryo. (c) Transverse histological section through the region of a morphologically visible ink mark. A 'plug' of ink particles is present in the mid-dorsal aspect of the neural tube. Section stained with haematoxylin and eosin. $d=$ dorsal aorta, $f l=$ fore limb bud, $g=$ hindgut, $h l=$ hind limb bud, $n=n o t o c h o r d$, $n c=$ neural canal, $n t=$ neural tube, $s=$ somites. Scale bars: (a) $0.5 \mathrm{~mm}$, (b) $0.25 \mathrm{~mm}$, (c) $50 \mu \mathrm{m}$. 
spina bifida, shows a male preponderance as in humans. $^{2}$

LEVEL OF SPINA BIFIDA IN $C T / C T$ EMBRYOS

Thirty-four $c t / c t$ embryos with spina bifida were studied, comprising a developmental series ranging from the earliest stage at which spina bifida can be recognised ( 32 to 35 somites) to the completion of body axis formation ( 60 somites). The rostral end of the spina bifida varied in position from somite 26 to 33 , with more than $90 \%$ of cases falling between the 27 th and 32nd somites (fig 1). The position of the caudal end of the spina bifida was much more variable. In 20 embryos, open neural folds extended throughout the caudal region of the embryo, a condition that was seen predominantly in younger embryos but also occurred in a minority of the more advanced embryos. In the other 14 embryos, the spina bifida did not extend to the tail tip. In these cases, the spina bifida abutted caudally onto a region of closed neural tube that extended to the tip of the developing tail. The position of this junction between spina bifida and closed neural tube was highly variable, ranging from somite 31 to 54 (fig 1).

Tail flexion defects (CT in fig 1) occurred in the majority of the more advanced embryos and, in addition, a number of these embryos had 'proximodistal fusion defects' ( $x$ in fig 1). In these cases, the recurved nature of the tail flexion defect appeared to have brought the dorsal surfaces of two regions of open spina bifida into direct contact, with the result that fusion had occurred secondarily between the apices of the open neural folds.

\section{SOMITE LEVEL OF POSTERIOR NEUROPORE CLOSURE}

The posterior neuropore closes during development at a level of the embryonic axis flanked by unsegmented, presomitic mesoderm. In order to determine the somite level of neuropore closure, we marked the position of the posterior neuropore, by injecting Indian ink into normal mouse embryos in which neuropore closure was imminent, and then culturing the injected embryos in vitro for 24 hours until somite formation had progressed caudally to the level of the neuropore. Of 28 embryos injected with Indian ink, 25 proved to have open posterior neuropores and were cultured further. After culture, 10 of the injected embryos had normal tail bud regions in which somites caudal to the hind limb bud were clearly visible (that is, the embryos had at least 31 somites) whereas the other 15 embryos had less well developed caudal regions with no somites caudal to the hind limb bud. Growth and development of these latter embryos appeared to have been adversely affected by constriction of the torn amnion and yolk sac following ink injection.
Of the 10 embryos with more than 31 somites, nine had prominent ink marks in the roof of the $\stackrel{5}{?}$ neural tube (fig $2 a, b$ ), in all cases within the region flanked by somites. The position of the ink marks in the nine normally developed embryos (table 2) indicated that the posterior neuropore had closed $\stackrel{\mathbb{Q}}{\Omega}$ between the levels of the 32nd and 34th somites, \& that is, distal to the rostral end of the spina bifida in the majority of $c t / c t$ embryos.

Transverse histological sections of these embryos showed that, in the region of the morphologically visible ink mark, ink particles formed a large 'plug' within the roof of the closed neural tube (fig 2c). At more rostral levels, ink was present only in the lumen of the neural tube. It appears, therefore, that injected ink is trapped by the closing neural folds at the posterior neuropore and becomes incorporated into the roof plate of the neural tube, thus providing a marker for the site of posterior neuropore closure.

\section{Discussion}

A knowledge of the precise level of closure of the posterior neuropore is critical if we are to determine whether lumbosacral spina bifida arises in mammals from a defect of primary or secondary neurulation. Table 3 shows the level of posterior neuropore closure in embryos of the three mammalian species studied to date. In the human and mouse the neuropore closes within the future sacral region, whereas in the hamster closure occurs at an earlier

TABLE 2 The position of closure of the posterior neuropore in Indian ink marked embryos.

\begin{tabular}{lll}
\hline Embryo No & $\begin{array}{l}\text { No of somites } \\
\text { present }\end{array}$ & $\begin{array}{l}\text { Somite level } \\
\text { of ink mark }\end{array}$ \\
\hline 1 & 33 & $32-33$ \\
2 & 35 & $33-34$ \\
3 & 35 & $32-33$ \\
4 & 33 & $32-33$ \\
5 & 33 & 32 \\
6 & 34 & 32 \\
7 & 35 & 34 \\
8 & 35 & $33-34$ \\
9 & 35 & 34 \\
Mean (SE) & $34 \cdot 2(0 \cdot 3)$ & $32 \cdot 9(0 \cdot 3)$ \\
\hline
\end{tabular}

TABLE 3 Level of posterior neuropore closure in mammals.

\begin{tabular}{|c|c|c|c|c|}
\hline Species & $\begin{array}{l}\text { Somite } \\
\text { stage }\end{array}$ & $\begin{array}{l}\text { Somite } \\
\text { level }\end{array}$ & $\begin{array}{l}\text { Spinal } \\
\text { level }\end{array}$ & Reference \\
\hline Human & 25 & 31 & $\begin{array}{l}\text { 2nd } \\
\text { sacral }\end{array}$ & 17 \\
\hline Mouse & $27-29$ & $32-34$ & $\begin{array}{l}\text { 2nd-4th } \\
\text { sacral }\end{array}$ & $\begin{array}{l}\text { 6. } 12,24 \text {, } \\
\text { present study }\end{array}$ \\
\hline Hamster & $21-22$ & $26-28$ & $\begin{array}{l}\text { 2nd-3rd } \\
\text { lumbar }\end{array}$ & 25 \\
\hline
\end{tabular}


stage of development, the posterior neuropore being situated in the lumbar region. The positioning of posterior neuropore closure in the future sacral region of human embryos (table 3 , based on the study of Muller and $\mathrm{O}^{\prime}$ Rahilly ${ }^{17}$ ) differs from the upper lumbar position suggested previously by Lemire et al. ${ }^{18}$ The reason for this discrepancy is that Muller and O'Rahilly ${ }^{17}$ made allowance for the length of unsegmented mesoderm that intervenes between the closing posterior neuropore and the most recently formed somite (number 25 ), whereas Lemire et al ${ }^{18}$ did not.

In the present study, we found that the rostral end of the spina bifida in ct/ct embryos (somites 27 to 32) is situated at a more cranial level than the position of closure of the posterior neuropore (somites 32 to 34 ) suggesting that spina bifida originates in $c t / c t$ embryos during primary neurulation. This conclusion is consistent with previous findings that spinal NTD, including open spina bifida, result from delayed closure of the posterior neuropore in $c t / c t$ embryos. ${ }^{12} 13$ An alternative possibility, that spina bifida arises initially in the region of secondary neurulation and then spreads in a retrograde fashion, caudocranially, to affect the region rostral to the previously closed neuropore, seems unlikely. In the present study, a region of open neural folds was present even in the least advanced embryos with 32 to 35 somites, in which secondary neurulation was only just beginning.

Does our conclusion with regard to the origin of lumbosacral spina bifida in $c t / c t$ embryos apply also to humans? The sacral position of posterior neuropore closure (table 3), taken together with the lumbosacral position of most cases of lower spinal NTD in humans, ${ }^{2}$ argues strongly that in human embryos, as in the $c t$ mouse, lower spina bifida is predominantly a defect of posterior neuropore closure.

Notwithstanding our conclusion regarding the origin of human and mouse lower spina bifida, it is clear from the study of $c t / c t$ embryos that secondary neurulation is also disturbed in many cases of lower spina bifida. It seems likely that a persistently open neuropore 'entrains' the secondary neurulation process to yield a bifid caudal neural tube. Then, as development proceeds, abnormal canalisation either continues throughout the caudal region or, at almost any time thereafter, may revert back to normal with formation of a closed neural tube. We have no evidence, however, that an open spina bifida can result solely from defective secondary neurulation, in the absence of a persistently open posterior neuropore.

Our study has confirmed the finding of Embury et $a l^{8}$ that exencephaly affects mainly female $c t / c t$ embryos whereas spinal NTD, taken together, show a sex ratio near to unity. Among embryos with spinal NTD (and no exencephaly), however, we find that open spina bifida affects males more than females whereas the converse is true of tail flexion defects alone. Thus, NTD in the $c t$ mouse appear to parallel the human condition even more closely than was previously thought, although the male preponderance in $c t / c t$ embryos is less marked than in humans. ${ }^{2}$

What is the embryological mechanism whereby defective neural folding in the cranial region affects mainly females and in the lower spine more often affects males? A clue has come from our finding that growth retardation of $c t / c t$ embryos both in vivo and in vitro leads to a reduction in the incidence of spinal NTD, with spina bifida being almost completely prevented. ${ }^{19}$ Studies of the cell cycle in $c t / c t$ embryos have shown an abnormally reduced rate of cell proliferation affecting the hind gut endoderm and notochord, but not the neuroepithelium, in the posterior neuropore region of embryos developing spinal NTD.$^{20}$ Growth retardation of $c t / c t$ embryos in vitro reduces the proliferative rate of neuroepithelium to a greater extent than gut endoderm and notochord. ${ }^{19}$ Thus, embryonic growth retardation serves to counteract the genetically determined cell proliferation imbalance thereby leading to normalisation of spinal neurulation. This preventive effect of growth retardation on spinal NTD contrasts with the exacerbating effect of growth retarding influences on the development of exencephaly in $c t / c t$ mice, ${ }^{21} 22$ an effect whose cellular basis is not understood.

From the preceding discussion, it is clear that the processes of primary neurulation in the cranial and lower spinal region of the mouse embryo differ fundamentally in their response to growth retarding influences on the embryo. Female $c t / c t$ embryos are known to be developmentally retarded compared with males at the onset of neurulation ${ }^{23}$ and, for this reason, may be more likely to develop exencephaly than males. ${ }^{2}$ On the other hand, later in development when the posterior neuropore is closing, growth and developmental retardation should actually protect female embryos against developing spinal NTD, in agreement with the observation that lower spinal NTD are more common in males ${ }^{2}$ (table 1). We conclude that fundamental differences in the mechanisms of primary neurulation in the cranial and lower spinal regions of mammalian embryos can account for the differences observed in sex incidence of NTD affecting these levels of the body axis.

We thank Dr M J Seller and Professor R L Gardner 
for commenting on the manuscript. The early part of this work was supported by grants from the Mental Health Foundation, Action Research for the Crippled Child, and the Association for Spina Bifida and Hydrocephalus. The later part of the work was supported by the Imperial Cancer Research Fund.

\section{References}

1 Carter CO. Clues to the aetiology of neural tube malformations. Dev Med Child Neurol 1974;16(suppl 32):3-15.

2 Seller MJ. Neural tube defects and sex ratios. Am J Med Genet 1987;26:699-707.

${ }^{3}$ Criley BB. Analysis of the embryonic sources and mechanisms of development of posterior levels of chick neural tubes. J Morphol 1969;128:465-501.

4 Jelinek R, Seichert V, Klika E. Mechanism of morphogenesis of caudal neural tube in the chick embryo. Folia Morphol (Praha) 1969;17:355-67.

${ }^{5}$ Lemire RJ. Variations in development of the caudal neural tube in human embryos (Horizons XIV-XXI). Teratology 1969;2: 361-70.

${ }^{6}$ Schoenwolf GC. Histological and ultrastructural studies of secondary neurulation of mouse embryos. Am J Anat 1984;169: 361-74.

7 Dryden RJ. Duplication of the spinal cord; a discussion of the possible embryogenesis of diplomyelia. Dev Med Child Neurol 1980;22:234-43.

${ }^{8}$ Embury S, Seller MJ, Adinolfi M, Polani PE. Neural tube defects in curly tail mice. I. Incidence, expression and similarity to the human condition. Proc $R$ Soc Lond [Biol] 1979;206: 85-94.

${ }^{9}$ Seller MJ, Adinolfi M. The curly-tail mouse: an experimental model for human neural tube defects. Life Sci 1981;29:1607-15.

10 Copp AJ. Teratology and experimental embryology: the pathogenesis of neural tube defects. In: Warshaw JB, ed. The biological basis of reproductive and developmental medicine. New York: Elsevier Biomedical, 1983:155-78.

"Seller MJ. Neural tube defects: cause and prevention. In: Adinolfi M, Benson PF, Gianelli F, Seller MJ, eds. Paediatric research. A genetic approach. London: Spastics International Medical Publications, 1982:197-211.

12 Copp AJ, Seller MJ, Polani PE. Neural tube development in mutant (curly tail) and normal mouse embryos: the timing of $\overrightarrow{\bar{F}}$ posterior neuropore closure in vivo and in vitro. $J$ Embryol Exp Morphol 1982;69:151-67.

13 Copp AJ. Relationship between timing of posterior neuropore closure and development of spinal neural tube defects in mutant (curly tail) and normal mouse embryos in culture. J Embryol Exp Morphol 1985;88:39-54.

${ }^{14}$ Gruneberg H. Genetical studies on the skeleton of the mouse. VIII. Curly tail. J Genet 1954;52:52-67.

${ }^{15}$ Hogan B, Costantini F, Lacy E. Manipulating the mouse embryo. New York: Cold Spring Harbor Laboratory, 1986.

${ }^{16}$ New DAT, Coppola PT, Terry S. Culture of explanted rat embryos in rotating tubes. J Reprod Fertil 1973;35:135-8.

17 Muller F, O'Rahilly R. The development of the human brain, the closure of the caudal neuropore, and the beginning of secondary neurulation at stage 12. Anat Embryol (Berl) 1987 176:413-30.

${ }^{18}$ Lemire RJ, Loeser JD, Leech RW, Alvord EC. Neurulation. In: Normal and abnormal development of the human nervous system. New York: Harper and Row, 1975:71-83.

19 Copp AJ, Crolla JA, Brook FA. Prevention of spinal neural tube defects in the mouse embryo by growth retardation during neurulation. Development 1988;104:297-303.

20 Copp AJ, Brook FA, Roberts HJ. A cell type-specific defect of cell proliferation in mutant mouse embryos developing spina neural tube defects. Development 1988;104:285-95.

${ }^{21}$ Seller MJ, Perkins KJ. Effect of hydroxyurea on neural tube defects in the curly-tail mouse. J Craniofac Genet Dev Biol 1983;3:11-7.

22 Seller MJ, Perkins KJ. Effect of mitomycin C on the neural tube defects of the curly-tail mouse. Teratology 1986;33:305-9.

${ }^{23}$ Seller MJ, Perkins-Cole KJ. Sex difference in mouse embryonic development at neurulation. J Reprod Fertil 1987;79:159-61.

${ }^{24}$ Sakai Y. Neurulation in the mouse. I. The ontogenesis of neural segments and the determination of topographical regions in a central nervous system. Anat Rec 1987;218:450-7.

${ }^{25}$ Shedden PM, Wiley MJ. Early stages of development in the caudal neural tube of the Golden Syrian Hamster (Mesocricetus auratus). Anat $\operatorname{Rec} 1987: 219: 180-5$.

Correspondence to Dr A J Copp, Medical Embryology Laboratory, Imperial Cancer Research Fund, Developmental Biology Unit, Zoology/Psychology Building, South Parks Road, Oxford OX1 3PS. 